\title{
Особенности исследования состояния
}

\section{связующего около частиц наполнителя в Эластомерных композитах с помощью атомно-силового микроскопа}

\author{
О. К. Гаришин ${ }^{a}$, Р. И. Изюмов ${ }^{a, b}$, А. Л. Свистков ${ }^{a, b}$

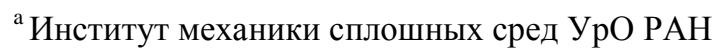 \\ 614013, Пермь, ул. Академика Королева, 1 \\ email: gar@icmm.ru \\ ${ }^{\mathrm{b}}$ Пермский государственный национальный исследовательский университет \\ 614990, Пермь, ул. Букирева, 15 \\ email: izumov@icmm.ru
}

\begin{abstract}
Атомно-силовая микроскопия (АСМ) является одним из наиболее перспективных методов исследования структуры материалов на микро и наноуровне, а также их локальных физикомеханических свойств. Поскольку получаемые с помощью АСМ экспериментальные данные малоинформативны, требуется их дальнейшая теоретическая расшифровка с привлечением различных математических и физических моделей. Кроме того, существует серьезная проблема оценки точности полученных на АСМ результатов, в частности, при исследовании материалов с сильно выраженной механической структурной неоднородностью. К таким материалам относятся рассматриваемые в данной работе эластомеры, наполненные жесткими дисперсными частицами. В статье приводятся методика математической обработки таких экспериментальных данных, позволяющая существенно повысить точность их расшифровки. В ее основе лежит использование трех критериев обработки полученной экспериментальной информации. Первый критерий «рельефа наноуровня» позволяет выделить из полученной при сканировании поверхности рельеф с объектами высокой кривизны. Именно на нем хорошо видны выступающие на поверхности частицы наполнителя (обычно закрытые тонкой пленкой эластомерного связующего, известного в литературе как связанный каучук). С помощью второго критерия «адгезионного отклонения» которого можно выделять частицы наполнителя по изменению адгезионных сил между зондом АСМ и поверхностью образца. Третий критерий «индентационной податливости», предназначен для определения на основе данных о жесткости частиц наполнителя их местоположения. Все эти критерии были использованы при расшифровке данных сканирования на АCM поверхности дисперсно наполненного эластомерного композита. Проведена оценка точности экспериментальных результатов на базе сравнения данных, полученных при прямом и обратном горизонтальном ходе зонда АСМ при сканировании одного и того же участка поверхности.
\end{abstract}

\section{Features of the study of binder state near the filler particles in elastomeric composites using an atomic-force microscope}

\author{
O. K. Garishin ${ }^{a}$, R. I. Izyumov ${ }^{a, b}$ A. L. Svistkov ${ }^{a, b}$
}

(С) Гаришин О. К., Изюмов Р. И., Свистков А. Л., 2018 


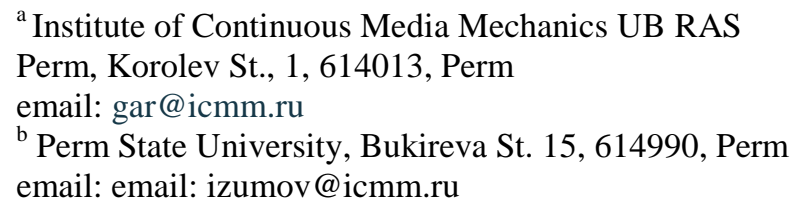

Atomic force microscopy (AFM) is one of the most promising methods for investigating the structure of materials at the micro and nanoscale levels, as well as their local physical-mechanical properties (which, as already known, can be very different from macroproperties). The experimental data obtained with the help of AFM are not very informative in themselves. Therefore, their further theoretical interpretation with the use of various mathematical and physical models is required. In addition, there is also a serious problem of assessing the accuracy and reliability of the results obtained for AFM. This is especially pronounced when studying materials with a strong mechanical structural heterogeneity. Such materials include considered in this paper elastomers, filled with rigid dispersed particles. The article presents a technique for mathematical processing of such experimental data, which allows to significantly improve the accuracy of their interpretation. It is based on the use of three criteria for filtering and processing the initially obtained experimental information. The first criterion of the "relief of the nanoscale" makes it possible to extract a relief with objects of low curvature from the overall picture obtained. It is on it that the filler particles protruding on the surface are clearly visible (usually covered with a thin film of elastomeric binder, known in the literature as bound rubber). The second is "adhesion deflection" criterion, by means of which it is possible to isolate the filler particles by changing the adhesion forces between the AFM probe and the sample surface. The third criterion of "indentational compliance", designed to determine the location and stiffness of the filler particles. All these criteria were used to decode the AFM scanning data of the surface of disperse filled elastomeric composite. Optimal conditions for the application of each of the criteria were determined from the analysis of the results obtained. Also, the accuracy of the experimental results was estimated on the basis of a comparison of the data obtained with the forward and reverse horizontal motion of the AFM probe when scanning the same surface area.

Keywords: atomic force microscopy; elastomers; nanofiller; nanostructure

Received 08.11.2017; accepted 25.01.2018

doi: 10.17072/1994-3598-2018-1-36-45

\section{1. Введение}

Одним из приоритетных направлений современного материаловедения является создание новых наноструктурированных материалов с улучшенными эксплуатационными свойствами (это так называемые нанокомпозиты). Решение этой задачи невозможно без глубоких фундаментальных знаний о внутреннем строении таких материалов и их локальных физико-механических свойствах на микро-, мезо- и наноуровне. Научившись эффективно управлять происходящими на этих масштабах процессами и явлениями, можно целенаправленно создавать материалы с принципиально новыми потребительскими качествами, недостижимыми в рамках использования традиционных технологий.

На сегодняшний день одним из наиболее перспективных инструментов для таких исследований является атомно-силовая микроскопия (АСМ) [15]. Ее основное преимущество по сравнению с традиционной электронной микроскопией состоит в том, что атомно-силовой микроскоп позволяет получать информацию не только о топологии внутреннего строения материала, но и о его ло- кальных физических свойствах. Еще в 1986 г. Герд Бининг, Келвин Куэйт и Кристофер Гербер [6] получили Нобелевскую премию за изобретение сканирующего атомно-силового микроскопа. В настоящее время их изобретение широко используется в самых разных областях современной науки - физике, химии, биологии и т.д. АСМ успешно применяется и в материаловедении при исследовании морфологии и локальных физикомеханических свойств материала на наноструктурном уровне (т.е. на масштабах, когда нужно учитывать эффекты, связанные с особенностями молекулярного строения вещества, хотя сам материал еще можно считать сплошной средой). Сегодня уже хорошо известно, что физические свойства наночастиц и кластеров, определяемые их чрезвычайно высокой удельной поверхностью, могут очень существенно отличаться от макроскопических характеристик [7].

С помощью АСМ ученые могут определять наноструктурные локальные упругие модули [815], параметры упрочнения [16, 17], ползучести [18], остаточные напряжения [19]. Данные технологии позволяют непосредственно наблюдать и количественно оценивать такие наномасштабные 
явления, как появление дислокаций, возникновение сдвиговой нестабильности, фазовые переходы и многие другие явления, недоступные для ранее известных технологий [20-24].

В основе работы АСМ лежит силовое взаимодействие между исследуемой поверхностью и консольной балкой (кантилевером) с острым кремниевым щупом на свободном конце. Как правило, этот щуп (зонд) имеет форму конуса со скругленной вершиной. Длина балки составляет около 100200 мкм, высота конуса 1-3 мкм. Радиус вершины зонда (который и определяет разрешающую способность прибора) у современных кантилеверов варьируется от 1 до 50 нанометров. Сила, действующая на зонд со стороны поверхности, приводит к изгибу консоли. Регистрируя величину изгиба, можно контролировать силу взаимодействия щупа с исследуемым объектом. В современных микроскопах для этого используются оптические методы. Оптическая система АСМ юстируется таким образом, чтобы излучение полупроводникового лазера фокусировалась на консоли зондового датчика, а отраженный луч попадал на регистрирующий фотодиод. Достаточно полное популярное описание принципов работы АCM можно найти, например, в [25, 26].

Особенно успешно методы наноиндентации развиваются в науке о полимерах. Большинство полимеров, как правило, намного мягче, чем материал зонда АСМ, что позволяет последнему достаточно глубоко проникать внутрь образца при контакте. В результате, исследуя процесс внедрения зонда в образец, можно получать уникальные сведения о механических свойствах материала на наноструктурном уровне.

В процессе эксперимента зонд АСМ сканирует выбранную поверхность образца. Получаемые при этом данные представляют собой зависимости между координатами точек сканирования $(x, y)$, вертикальной координатой основания зонда $(z)$ и отклонением конца кантилевера от свободного состояния $d$, по которому вычисляется сила, действующая на зонд $F$ [26]. Эти результаты сами по себе (без дополнительных знаний о предмете исследований) малоинформативны, поэтому требуется их дальнейшая теоретическая расшифровка с привлечением различных математических и физических методов.

Кроме того, существует серьезная проблема оценки точности полученных на АСМ результатов. Насколько мы можем судить из анализа известных публикаций [27-33], в большинстве работ основное внимание уделяется исследованию влияния на результат сканирования таких факторов, как форма зонда, воздействие зонда на образец, влияние влажности, нелинейные эффекты пьезоэлектриков. В данной работе рассматриваются специфические эффекты, возникающие при сканировании дисперсно наполненных эластомеров с помощью АCM, обсуждается вопрос надёжности получаемых результатов и предлагается новая методика решения выявленных проблем.

\section{2. Критерии определения участков поверхности образца, в которых около границы материала с воздухом располагаются частицы наполнителя}

\section{1. Критерий выделения участков поверхности с помощью рельефа наноуровня}

Поверхность исследуемых с помощью атомносиловой микроскопии образцов представляет собой сложный рельеф. Его можно представить как сумму двух рельефов [34]. Это медленно меняющийся рельеф малой кривизны (поверхность макроуровня материала) и рельеф высокой кривизны (поверхность наноуровня материала). Именно на нем видны выступающие частицы наполнителя, обычно закрытые тонкой пленкой эластомерного связующего, известного в литературе как связанный каучук.

Выделить рельеф наноуровня можно с помощью интегрального фильтра, определяемого следующим преобразованием:

$$
\mathrm{z}_{\text {nano }}(x, y)=\int_{S} \varphi(r) \mathrm{z}\left(x_{1}, y_{1}\right) d S,
$$

где $z_{\text {nano }}$ - высота расположения точки наноуровня над горизонтальной поверхностью; $z$ - координата по вертикальной оси текущей точки на области интегрирования $S ; x, y$ - координаты искомой точки поверхности наноуровня на горизонтальной плоскости; $x_{1}, y_{1}$ - координаты текущей точки в области интегрирования $S$ на горизонтальной плоскости. Ядро интегрального оператора имеет вид

$$
\begin{aligned}
& \varphi(r)=\delta(r)-C H(L-r), \\
& r=\sqrt{\left(x-x_{1}\right)^{2}+\left(y-y_{1}\right)^{2}},
\end{aligned}
$$

где $\delta(x)$ - функция Дирака; $H(x)$ - функция Хэвисайда; $L$ - характерный размер, используемый для выделения рельефа наноуровня. Константа $C$ определяется из условия нормировки

$$
\int_{S} \varphi(r) d S=1 .
$$

В дальнейшем будут использованы экспериментальные данные, полученные на квадратной области поверхности образца, сторона которой равна 1.5 мкм (рис. 1, а). Однако сторона квадратной области, используемой для исследования, будет немного меньше. Это связано с тем, что для применения интегрального фильтра с целью получения рельефа наноуровня необходимо исключить из рассмотрения точки, расположенные от границы ближе расстояния $L$. Кроме этого, размеры области будут уменьшены в соответствии с необхо- 


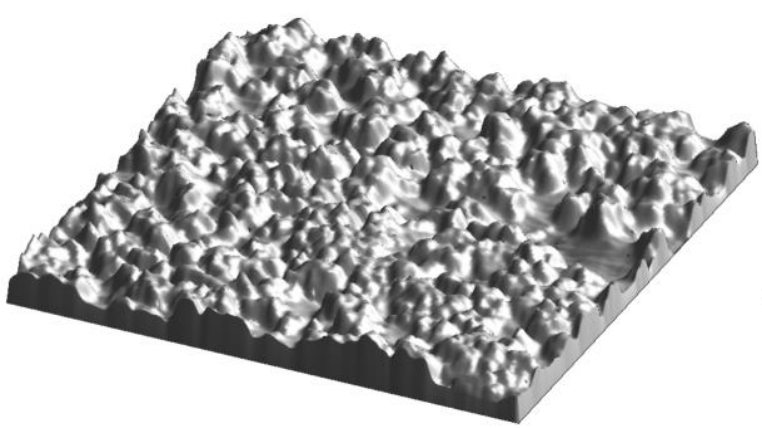

(a)

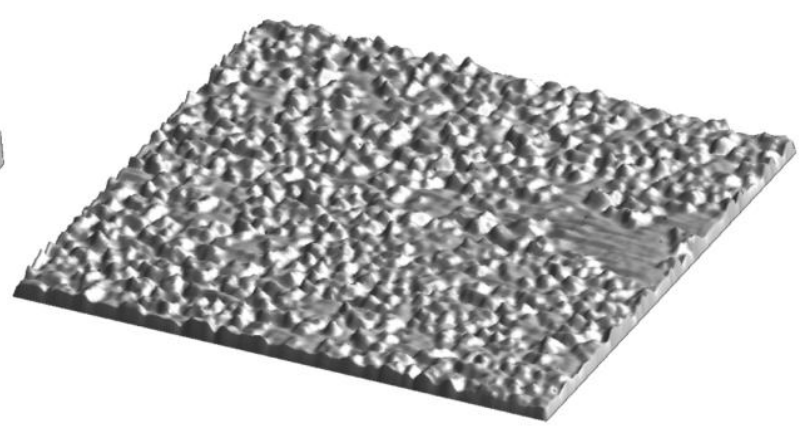

(б)

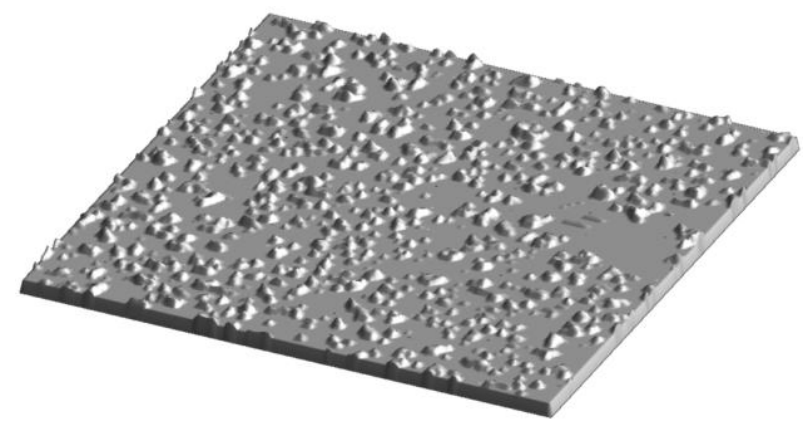

(B)

Рис. 1. Рельеф исследуемого участка поверхности образиа (а) и получаемые из него, после преобразования с помощью интегрального фильтра, две новые поверхности: б - поверхность объектов высокой кривизны (неровности наноуровня) и в - поверхность вершин неровностей наноуровня

димостью количественной оценки точности экспериментальных данных. Об этом речь пойдет позже. В результате использования фильтра получается рельеф, показанный на рисунке 1, б. В расчетах был выбран характерный размер $L=25 \mathrm{HM}$.

Следующим шагом является избавление от шумов около нулевой координаты на рельефе поверхности наноуровня и выделение вершин на этом рельефе. Для этого отсекаем горизонтальной плоскостью части рельефа, у точек которых высоты расположены ниже высоты $C_{\mathrm{z}}=0.03 \mathrm{~L}$ (рис. 1, в). Выступающие над этой плоскостью вершины определяют участки, где ожидается расположение частиц наполнителя около границы материала с воздухом. Нахождение вероятного расположения частиц на основе анализа высот поверхности наноуровня $z_{\text {nano }}$ будем называть далее первым критерием. Формулируется он следу- ющим неравенством:

$$
z_{\text {nano }}<C_{\mathrm{z}}
$$

\section{2. Критерий выделения участков поверхности с помощью параметров кривой индентирования материала}

В литературе для получения информации о механических свойствах материала, получаемых в режимах наноиндентирования, используются параметры, показанные на рис. 2. Это смещение крепления кантилевера $\Delta z$, глубина внедрения зонда в материал $u$, отклонение зонда относительно основания кантилевера $d$ (которое отсчитывается от ненагруженного состояния), удаление зонда от точки наибольшего его внедрения в материал $\gamma$ (который в англоязычной литературе называется «separation»). Направление отсчета соответствующих параметров от нулевого значения показано стрелками на рис. 2 .
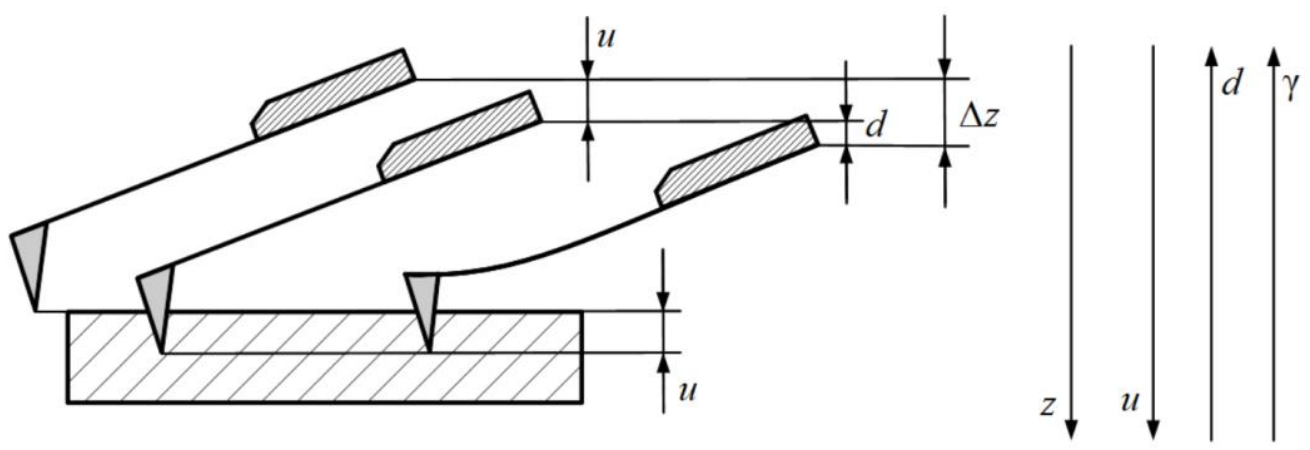

Рис. 2. Иллюстрация, показывающая физический смысл используемых параметров в задачах наноиндентирования и направление осей для отсчета соответствующих величин 
Вторым критерием нахождения участков поверхности, у которых около границы материала расположены частицы наполнителя, будет минимальное значение отклонение кончика зонда $d_{\mathrm{A}}$, взятое с противоположным знаком (рис. 3). Достигается оно на обратном ходе зонда, когда происходит извлечение его из материала. Это момент, когда начинается отрыв или сползание прилипшего к зонду эластомера при нахождении его над поверхностью образца.

Параметр $d_{\mathrm{A}}$ в дальнейшем будем называть адгезионным отклонением. Нетрудно понять, почему он позволяет судить о наличие частиц наполнителя около поверхности образца. Мы рассматриваем эластомерные материалы с активными нанонаполнителями. У них высокая энергия взаимодействия с молекулами полимера. Это означает, что около частиц наполнителя имеется тонкий слой полимеpa. В литературе его называют связанным каучуком. Чем глубже погружается зонд в материал, тем большее количество эластомера налипает на него, и тем больше требуется усилие для отрыва зонда от полимера при отводе зонда от образца (рис. 4).

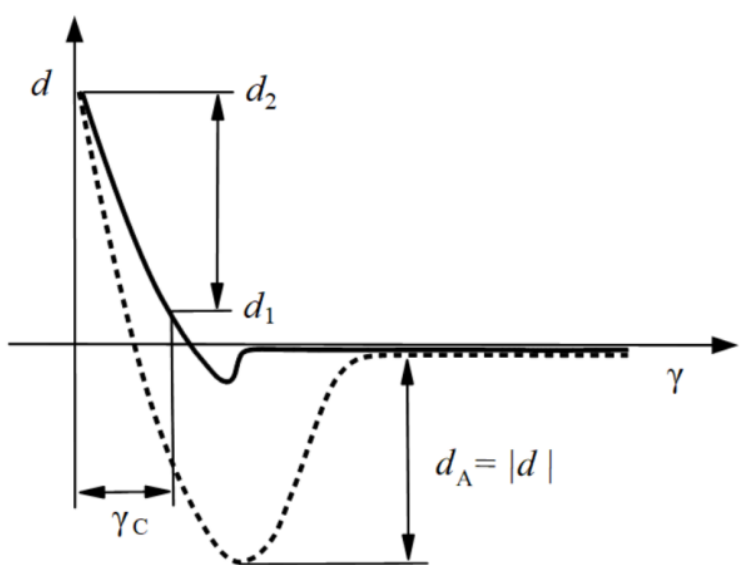

Рис. 3. Кривая наноиндентирования, определяющая зависимость отклонение зонда относительно основания кантилевера от параметра удаления зонда от точки его наибольшего внедрения в материал

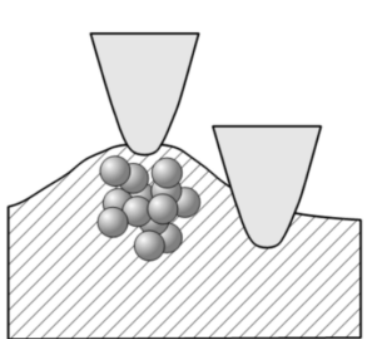

(a)

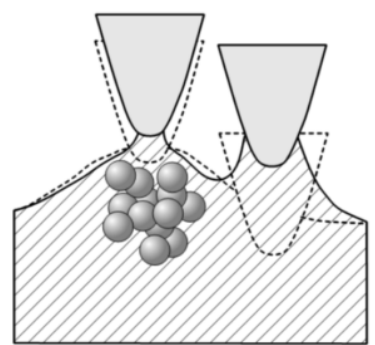

(б)
Рис. 4. Иллюстрации зависимости адгезионного отклонения $d_{\mathrm{A}}$ от величины максимального внедрения зонда в материал: $a$-соответствует максимальному внедрению зонда; б - моменту отрыва эластомера от зонда на обратном ходе
Поэтому в качестве второго критерия выделения частиц наполнителя целесообразно использовать условие

$$
d_{\mathrm{A}}<C_{\mathrm{A}} .
$$

Значение параметра $C_{\mathrm{A}}$ будем выбирать из условия, чтобы площадь проекций выделенных участков на горизонтальную плоскость по второму критерию совпадала с площадью проекций участков, выделенных по первому критерию.

Третьим критерием является индентационная податливость $\gamma_{\mathrm{c}}$. В англоязычной литературе её называют параметром «deformation». Определяется она следующим образом. Максимальное отклонение зонда относительно основания кантилевера обозначим параметром $d_{2}$ (рис. 3). Значение параметра выбиралось из условия $d_{1}=0.15 \cdot d_{2}$. Тогда разница между соответствующими значениями параметра удаления зонда от глубины наибольшего внедрения на прямом ходе (идёт погружение зонда в образец) представляет собой индентационную податливость $\gamma_{\mathrm{c}}=\gamma\left(d_{1}\right)-\gamma\left(d_{2}\right)$. По своему физическому смылу это часть перемещения зонда внутри материала. На данном интервале отличительной особенностью взаимодействия зонда с образцом является то, что основная работа идет на деформирование материала. В противооположность этому на меньшей глубине внедрения существенно проявляются другие явления. На силу, действующую на зонд, существенно виляет взаимодействие его с поверхностью образца (затраты энергии, идущей на формирования новой поверхности контакта, уменьшение площади контакта образца и зонда с воздухом и т.д.).

Важным моментом, на который следует обращать внимание, - это случай, когда зонд отскакивает от поверхности образца. Связано это с тем, что нами исследуется материал, в котором встречаются то очень жесткие области, то очень мягкие. Поэтому невозможно настроить атомносиловой микроскоп таким образом, чтобы с удовлетворительной точностью получалась экспериментальная информация на всей исследуемой поверхности. Вхождения зонда в материал происходит с достаточно большой скоростью. Поэтому возможны ситуации с отскоком зонда. Для таких точек получается отрицательное значение индентационной податливости $\gamma_{\mathrm{c}}$.

Если недалеко под тонким слоем эластомера находится частица наполнителя, то внедрение зонда в образец потребует значительного усилия. Поэтому в качестве третьего критерия выделения частиц наполнителя имеет смысл использовать неравенство

$$
\gamma_{\mathrm{c}}<C_{\gamma},
$$

в котором значение параметра $C_{\gamma}$ будем выбирать из условия, чтобы площадь проекций выделенных частиц на горизонтальную плоскость по второму 
критерию совпадала с площадью проекций частиц, выделенных по первому критерию.

\section{3. Точность экспериментальных данных, получаемых в режиме механического картирования}

Важную роль играет проверка точности экспериментальных данных и величин, которые получаются в результате их обработки. В первую очередь следует обратить внимание на карты рельефа поверхности, полученные в режимах trace и retrace. Они отличаются друг от друга. Режим trace - это прохождение зондом по выбранной области материала в одном направлении. Retrace - это получение экспериментальных данных при движении зонда по выбранной области в обратном направлении.

Чтобы добиться наилучшего совпадения, необходимо сместить карты относительно друг друга. Вероятно, это связано с тем, что для изменения направления движения зонда требуется некоторое время, в течение которого снимается информация о нескольких точках при движении в одном направлении и не учитывается при движении в противоположном. Поэтому для исследования нами выбрана часть поверхности, которая является общей для карт, полученных в режимах trace и retrace. Карты с параметрами адгезионного отклонения и индентационной податливости смещены так же, как и карты рельефа поверхности материала, и точно также взята для исследования общая область поверхности образца.

Дальнейшее исследование карт поверхности образца показало, что всегда остается заметное отличие в рельефах поверхности, полученных в режимах trace и retrace. На рис. 5 осуществлено сравнение областей материала, выделенных с помощью первого критерия в режимах trace и retrace. Но это отличие не столь значительное. Расхождение занимают около $6 \%$ площади выбранного участка поверхности образца.

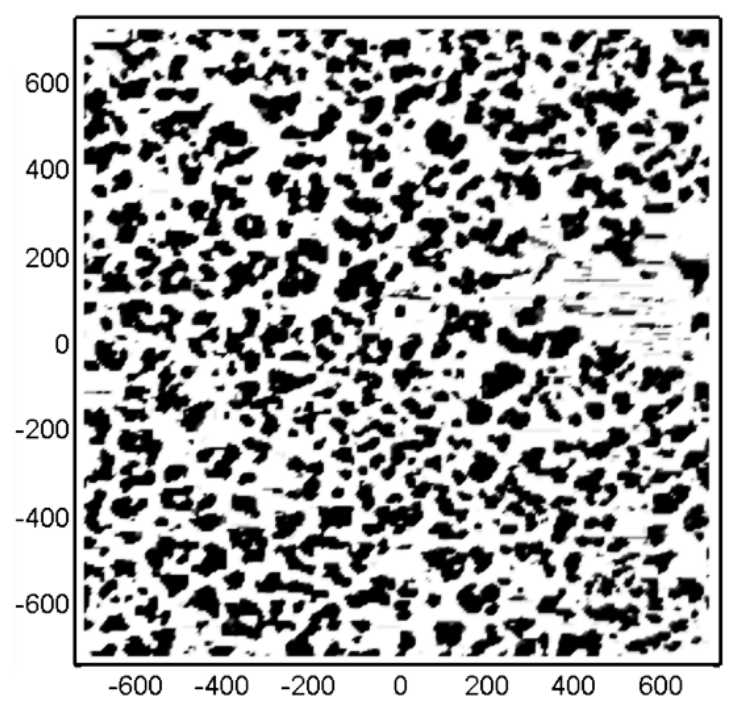

(a)

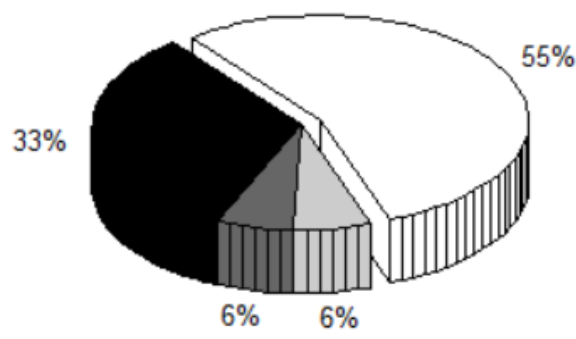

(б)

Рис. 5. Выделенные участки поверхности образца, у которых около границы материала расположены частицы наполнителя (а) и доли проекциий от участков поверхности (б): черный цุвет - участки, где одновременно выполняются критерии по высотам на картах, полученных в режимах trace и retrace; темно-серый цьвет - только в режиме trace; светло-серый извет - только в режиме retrace; бельй - ни в одном режиме

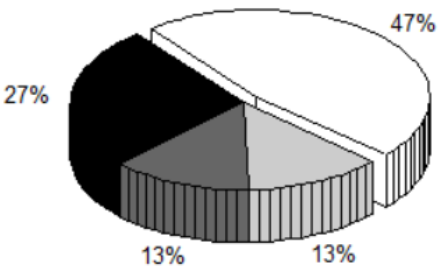

(a)

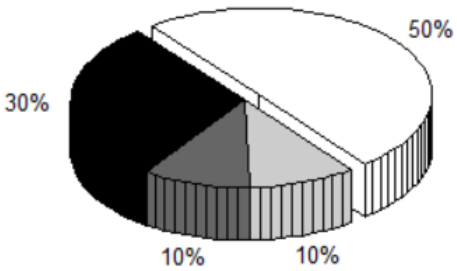

(б)

Рис. 6. Доли, которые занимают проекции участков материала, выделенные с помощью двух критериев: а-по высотам и индентационной податливости; б - по высотам и адгезионному отклонению. На диаграммах черный ияет соответствует - одновременному выполнению двух критериев; темносерый - только выполнению критерия по высотам; светло-серый - только второму критерию 


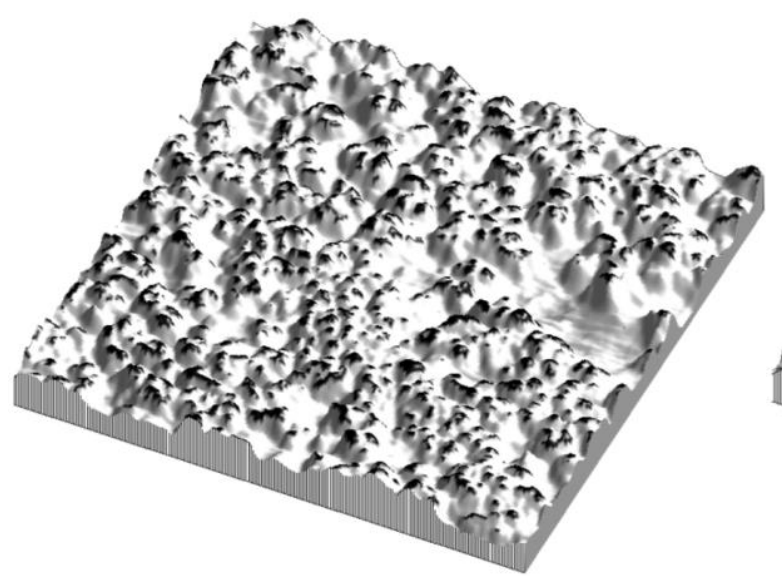

(a)

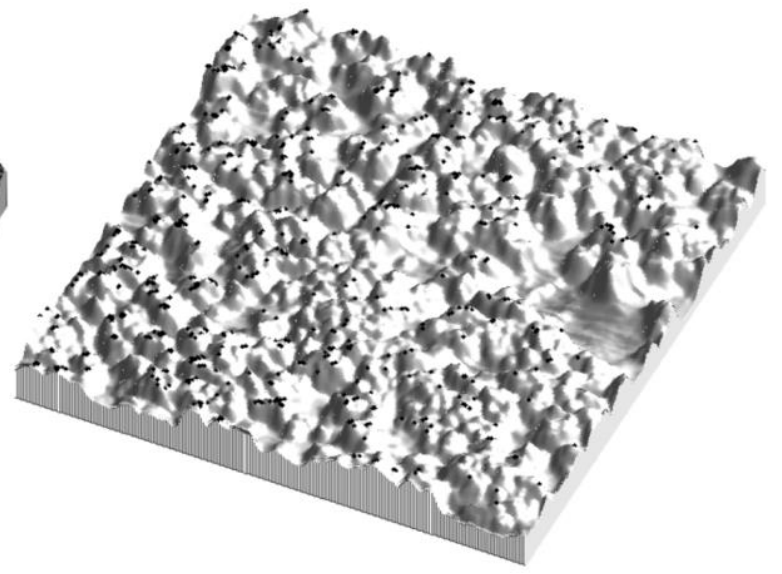

(б)

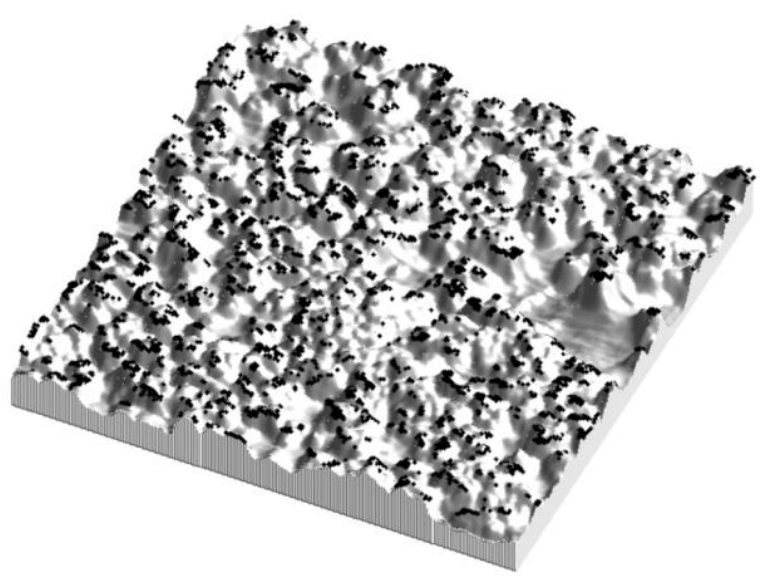

(B)

Рис. 7. Рельеф исследуемого участка поверхности образиа, на котором вылделены: : - черным иветом области, где выполняются одновременно три критерия расположения частиц наполнителя около поверхности материала; б - точки, где произошел отскок зонда от поверхности образиа; в - точки, в которых выполняются три критерия, и нет отскока зонда от поверхности образца

Значительно хуже получаются результаты, если осуществляется сравнение участков, выделенных с помощью разных критериев. Показанное на рис. 6 сравнение осуществлено для значений карт, являющихся средним арифметическим значений соответствующих параметров, полученных в режимах trace и retrace. Это параметры: высота рельефа наноуровня, адгезионное отклонение, индентационная податливость. Отличие в этом случае достигает 10 и более процентов.

Однако имеются участки поверхности образца, на которых одновременно выполняются все три критерия (рис. 7, а). Это позволяет уверенно полагать, что это именно те области поверхности материала, около которых гарантированно располагаются частицы наполнителя. Причем экспериментальные данные позволяют отметить возможную особенность взаимодействия зонда атомно-силового микроскопа с материалом. Речь идет о ситуациях, когда зонд ударяется о поверхность образца и отскакивает от него (рис. 7, б). В таких точках значение параметра «индентационная податливость» будет отрицательной. Расшифровка особенностей взаимодействия зонда с материалом требует в этом случае учета динамических эффектов, точность которых сложно определить. Поэтому, по нашему мнению, целесообразно ограничиться в дальнейшем анализом кривых индентирования только в областях, где выполняются одновременно три критерия, и нет отскока зонда от поверхности образца.

В рассматриваемом примере на таких выделенных участках средние значения адгезионного отклонения и индентационной податливости равны соответственно 10.3 нм и 1.5 нм. Среднеквадратичное отклонение значений адгезионного отклонения и адгезионной податливости равны 2.3 нм и 1.1 нм. Дальнейшая расшифровка этих величин требует использования математических моделей индентирования материала.

\section{4. Выводы}

Проведенные исследования показали, что обязательным условием при обработке данных атомно-силовой микроскопии о структуре и свойствах эластомерных нанокомпозитов является оценка точности получаемых результатов. Предложено выполнять такую оценку путем сравнения карт параметров, полученных в режимах trace и retrace. 
Выделение областей материала, в которых около поверхности образца располагаются частицы наполнителя, осуществлено с использованием критериев "рельеф наноуровня", "адгезионное отклонение" и "индентационная податливость". Установлено, что их применение приводит к выделению отличающихся участков поверхности образца. Тем не менее можно выделить такие участки поверхности, в которых выполняются одновременно все три критерия. На них целесообразно определять значения возможной толщины слоя эластомера, жесткости, силы взаимодействия с зондом и точность исследуемых величин.

Авторы статьи благодарят И. А. Морозова за помощь и предоставленные нам экспериментальные данные, и РФФИ за оказанную финансовую поддержку исследований в рамках проекта № 1608-00914.

\section{Список литературы}

1. Giessib F. J. AFM's path to atomic resolution // Materials Today. 2005. Vol. 8. N. 5. P. 32-41.

2. Butt H., Capella B., Kappl M. Force measurements with the atomic force microscope: Technique, interpretation and applications // Surface science reports. 2005. Vol. 59. P. 1-152.

3. Schuh C. A. Nanoindentation studies of materials // Materials Today. 2006. Vol. 9. N. 5. P. 32-40.

4. He Y., Geng Y., Yan Y. et al. Fabrication of nanoscale pits with high throughput on polymer thin film using afm tip-based dynamic plowing lithography // Nanoscale Research Letters. 2017. Vol. 12, 544. DOI: 10.1186/s11671-017-2319-y

5. Yan Y., Geng Y., Hu Z. Recent advances in AFM tip-based nanomechanical machining // International Journal of Machine Tools and Manufacture. 2015. Vol. 99. P. 1-18.

DOI: 10.1016/j.ijmachtools.2015.09.004

6. Binnig G., Quate C. F., Gerber C. Atomic force microscope // Physical Review Letters. 1986. Vol. 56. N. 9. P. 930-933.

7. Чвалун C. Н. Полимерные нанокомпозиты // Природа. 2000. Вып. 7. С. 22-30.

8. Vanlandingham M. R., McKnicht S. H., Palmese $G$. R. et al. Relating elastic modulus to indentation response using atomic force microscopy // Journal of Materials Science Letters. 1997. Vol. 16. P. 117-119.

9. Bhushan B. Handbook of micro-mano-tribology // Springer. 1999. 433 p.

10. Johnson L. L. Atomic force microscopy (AFM) for rubber // Rubber chemistry and technology. 2008.

11. Poon B., Rittel D., Ravichandran G. An analysis of nanoindentation in linearly elastic solids // International Journal of Solids and Structures. 2008. Vol. 45. N. 24. P. 6018-6033.

DOI: $10.1016 /$ j.ijsolstr.2008.07.021
12. Pettersson T., Hellwig J., Gustafsson P. J. et al. Measurement of the flexibility of wet cellulose fibers using atomic force microscopy // Cellulose. 2017. Vol. 24. N. 4139. DOI: 10.1007/s10570017-1407-6

13. Reggente M., Rossi M., Angeloni L. et al. Atomic force microscopy techniques for nanomechanical characterization: a polymeric case study // JOM. 2015. Vol. 67(849). DOI: 10.1007/s11837-0151340-9

14. Ding Y. H., Deng X. H., Jiang X. et al. Nanoscale mechanical characterization of PMMA by AFM nanoindentation: a theoretical study on the timedependent viscoelastic recovery // Journal of Materials Science. 2013. Vol. 48, 3479. DOI: 10.1007/s10853-013-7138-7

15. Chen A., Qian C., Chen Y. et al. Exploring the elastic behavior of core-shell organic-inorganic spherical particles by afm indentation experiments // Journal of Inorganic and Organometallic Polymers and Materials. 2014. Vol. 24, 1070. DOI: 10.1007/s10904-014-0098-9

16. Dao M., Chollacoop N., Van Vliet K. J. et al. Computational modeling of the forward and reverse problems in instrumented indentation // Acta Mater. 2001. Vol. 49. B. 19. P. 3899-3918.

17. Gadelrab K. R., Bonilla F. A., Chiesa M. Densification modeling of fused silica under nanoindentation // Journal of Non-Crystalline Solids. 2012. Vol. 358. N. 2. P. 392-398.

18. Fischer-Cripps A. C. Nanoindentation and indentation measurements // Material Science and Engineering. 2004. Vol. 44. P. 91-102.

19. Carlsson S., Larsson P. L. On the determination of residual stress and strain fields by sharp indentation testing. Part I: theoretical and numerical analysis // Acta Materialia. 2001. Vol. 49. N. 12. P. 2179-2191.

20. Fischer-Cripps A. C. Nanoindentation // Springer. 2002. 217 p.

21. Wong S., Haberl B., Williams J. S. et al. Phase transformation dependence on initial plastic deformation mode in Si via nanoindentation // Experimental Mechanics. 2017. Vol. 57, 1037. DOI: $10.1007 / \mathrm{s} 11340-016-0213-7$

22. Chrobak D., Kwang-Ho Kim, Kurzydłowski K. J. et al. Nanoindentation experiments with different loading rate distinguish the mechanism of incipient plasticity // Applied Physics Letters. 2013. Vol. 103, 072101. DOI: 10.1063/1.4818260

23. Zhang D., Wang X., Song W. et al. Analysis of crystallization property of LDPE/Fe3O4 nanodielectrics based on AFM measurements // Journal of Material Science: Materials in Electronics. 2017. Vol. 28, 3495. DOI: 10.1007/s10854-0165948-5

24. Yew Z. T., Olmsted P. D., Paci E. Free energy landscapes of proteins: insights from mechanical probes // Single-Molecule Biophysics. 2012. Vol. 146. P. 395-417. 
25. Головин Ю. И. Введение в нанотехнологию. М.: Машиностроение. 2003. 112 с.

26. Миронов В. Л. Основы сканирующей зондовой микроскопии. - Н. Новгород: изд-во Института физики микроструктур РАН. 2004. 115 с.

27. Филонов А., Яминский И. Обработка и анализ данных в сканирующей зондовой микроскопии: алгоритмы и методы // Наноиндустрия. 2007. Вып. 2. С. 32-34.

28. D'Acunto M., Dinelli F., Pingue P. Nanoscale rippling on polymer surfaces induced by AFM manipulation // Beilstein Journal of Nanotechnology. 2015. Vol. 6. P. 2278-2289.

DOI: $10.3762 /$ bjnano.6.234

29. Keryvin V., Charleux L., Bernard C. et al. The influence of indenter tip imperfection and deformability on analysing instrumented indentation tests at shallow depths of penetration on stiff and hard materials // Experimental Mechanics. 2017. Vol. 57, 1107. DOI: 10.1007/s11340-017-0267-1

30. Fischer-Cripps A. C. Nanoindentation. 2011. DOI 10.1007/978-1-4419-9872-9

31. Korayem A. H., Taghizadeh M., Abdi M. et al. Experimental analysis of rough surface topography and modifying the humidity effect in AFM images to improve the topography quality // International Journal of Advanced Manufacturing Technology. 2017. DOI: $10.1007 / \mathrm{s} 00170-017-0928-3$

32. Rana M. S., Pota H. R, Petersen I. R. Nonlinearity Effects Reduction of an AFM Piezoelectric Tube Scanner Using MIMO MPC // IEEE/ASME Transactions on Mechatronics. 2015. Vol. 20 (3). DOI: 10.1109/TMECH.2014.

33. Liu W., Cheng L., Hou Z., Tan M. An active disturbance rejection controller with hysteresis compensation for piezoelectric actuators // Intelligent Control and Automation (WCICA). 2016. DOI: 10.1109/WCICA.2016.757859

34. Ужегова Н. И., Свистков А. Л. Многоуровневый анализ рельефа поверхности образца, полученного методами атомно-силовой микроскопии // Вычислительная механика сплошных сред. 2016. Т. 9. Вып. 3. С. 366-374.

\section{References}

1. Giessib F. J. AFM's path to atomic resolution. $M a$ terials Today, 2005, vol. 8, no. 5, pp. 32-41.

2. Butt H. Force measurements with the atomic force microscope: Technique, interpretation and applications / H. Butt, B. Capella, M. Kappl. Surface science reports, 2005, vol. 59, pp. 1-152.

3. Schuh C. A. Nanoindentation studies of materials. Materials Today, 2006, vol. 9, no. 5, pp. 32-40.

4. He, Y., Geng, Y., Yan, Y. et al. Fabrication of nanoscale pits with high throughput on polymer thin film using afm tip-based dynamic plowing lithography. Nanoscale Res Lett, 2017, vol. 12(544). DOI: 10.1186/s11671-017-2319-y
5. Yan Y., Geng Y., Hu Z. Recent advances in AFM tip-based nanomechanical machining. Int J Mach Tool Manu, 2015, vol. 99, pp. 1-18. DOI: 10.1016/j.ijmachtools.2015.09.004

6. Binnig G., Quate C. F., Gerber C. Atomic force microscope. Phys. Rev. Lett, 1986, vol. 56, no. 9, pp. 930-933.

7. Chvalun S. N. Polimernyye nanokompozity. Priroda, 2000, no. 7, pp. 22-30. (In Russian).

8. Vanlandingham M. R., McKnicht S. H., Palmese G. R. et al. Relating elastic modulus to indentation response using atomic force microscopy. Journal of Materials Science Letters, 1997, vol. 16, pp. 117-119.

9. Bhushan B. Handbook of micro-mano-tribology. Springer, 1999, 433 p.

10. Johnson L. L. Atomic force microscopy (AFM) for rubber. Rubber chemistry and technology, 2008.

11. Poon B., Rittel D., Ravichandran G. An analysis of nanoindentation in linearly elastic solids. International Journal of Solids and Structures, 2008, vol. 45, no. 24, pp. 6018-6033.

DOI: $10.1016 /$ j.ijsolstr.2008.07.021

12. Pettersson T., Hellwig J., Gustafsson P. J. et al. Measurement of the flexibility of wet cellulose fibers using atomic force microscopy. Cellulose, 2017, vol. 24, no. 4139. DOI: 10.1007/s10570017-1407-6

13. Reggente M., Rossi M., Angeloni L. et al. Atomic force microscopy techniques for nanomechanical characterization: a polymeric case study. JOM, 2015, vol. 67, 849. DOI: 10.1007/s11837-0151340-9

14. Ding Y. H., Deng X. H., Jiang X. et al. Nanoscale mechanical characterization of PMMA by AFM nanoindentation: a theoretical study on the timedependent viscoelastic recovery. Journal of Material Science, 2013, vol. 48, 3479. DOI: 10.1007/s10853-013-7138-7

15. Chen A., Qian C., Chen Y. et al. Exploring the elastic behavior of core-shell organic-inorganic spherical particles by afm indentation experiments. Journal of Inorganic and Organometallic Polymers and Materials, 2014, vol. 24, 1070. DOI: 10.1007/s10904-014-0098-9

16. Dao M., Chollacoop N., Van Vliet K. J. et al. Computational modeling of the forward and reverse problems in instrumented indentation. Acta Mater, 2001, vol. 49, B. 19, pp. 3899-3918.

17. Gadelrab K. R., Bonilla F. A., Chiesa M. Densification modeling of fused silica under nanoindentation. Journal of Non-Crystalline Solids, 2012, vol. 358, no. 2, pp. 392-398.

18. Fischer-Cripps A. C. Nanoindentation and indentation measurements. Mater. Sci. Eng, 2004, vol. 44, pp. 91-102.

19. Carlsson S., Larsson P. L. On the determination of residual stress and strain fields by sharp indentation testing. Part I: theoretical and numerical anal- 
ysis. Acta Materialia, 2001, vol. 49. N.12, pp. 2179-2191.

20. Fischer-Cripps A. C. Nanoindentation. Springer, 2002. $217 \mathrm{p}$.

21. Wong S., Haberl B., Williams J. S. et al. Phase transformation dependence on initial plastic deformation mode in Si via nanoindentation. Experimental Mechanics, 2017, vol. 57, 1037. DOI: $10.1007 / \mathrm{s} 11340-016-0213-7$

22. Chrobak D., Kwang-Ho Kim, Kurzydłowski K. J. et al. Nanoindentation experiments with different loading rate distinguish the mechanism of incipient plasticity. Applied Physics Letters, 2013, vol. 103, 072101. DOI: $10.1063 / 1.4818260$

23. Zhang D., Wang X., Song W. et al. Analysis of crystallization property of LDPE/Fe3O4 nanodielectrics based on AFM measurements. Journal of Material Science: Materials in Electronics. , 2017, vol. 28, 3495. DOI: 10.1007/s10854-0165948-5

24. Yew Z. T., Olmsted P. D., Paci E. Free energy landscapes of proteins: insights from mechanical probes. Single-molecule Biophysics, 2012, vol. 146, pp. 395-417.

25. Golovin Yu. I. Vvedeniye $v$ nanotekhnologiyu. Moscow: Mashinostroyeniye, 2003. 112 p. (In Russian).

26. Mironov V. L. Osnovy skaniruyushchey zondovoy mikroskopii. N. Novgorod: izd-vo Instituta fiziki mikrostruktur RAN, 2004. 115 p. (In Russian).

27. Filonov A., Yaminskiy I. Obrabotka i analiz dannykh v skaniruyushchey zondovoy mikroskopii: algoritmy i metody. Nanoindustriya, 2007, Vol. 2. pp. 32-34. (In Russian).
28. D'Acunto M., Dinelli F., Pingue P. Nanoscale rippling on polymer surfaces induced by AFM manipulation. Beilstein Journal of Nanotechnology, 2015, vol. 6, pp. 2278-2289.

DOI: $10.3762 /$ bjnano.6.234

29. Keryvin V., Charleux L., Bernard C. et al. The influence of indenter tip imperfection and deformability on analysing instrumented indentation tests at shallow depths of penetration on stiff and hard materials. Experimental Mechanics, 2017, vol. 57, 1107. DOI: $10.1007 / \mathrm{s} 11340-017-0267-1$

30. Fischer-Cripps A. C. Nanoindentation, 2011. DOI 10.1007/978-1-4419-9872-9

31. Korayem A. H., Taghizadeh M., Abdi M. et al. Experimental analysis of rough surface topography and modifying the humidity effect in AFM images to improve the topography quality. International Journal of Advanced Manufacturing Technology, 2017. DOI: 10.1007/s00170-017-0928-3

32. Rana M. S., Pota H. R, Petersen I. R. Nonlinearity Effects Reduction of an AFM Piezoelectric Tube Scanner Using MIMO MPC. IEEE/ASME Transactions on Mechatronics, 2015, vol. 20 (3). DOI: 10.1109/TMECH.2014.

33. Liu W., Cheng L., Hou Z., Tan M. An active disturbance rejection controller with hysteresis compensation for piezoelectric actuators. Intelligent Control and Automation (WCICA), 2016. DOI: 10.1109/WCICA.2016.757859

34. Uzhegova N. I., Svistkov A. L. Multilevel analysis of the relief of a surface sample obtained by atomic force microscopy techniques. Computational Continuum Mechanics, 2016, vol. 9, no. 3, pp. 366-374 (In Russian)

Просьба ссылаться на эту статью в русскоязычных источниках следующим образом:

Гаришин О. К., Изюмов Р. И, Свистков А. Л. Особенности исследования состояния связующего около частиц наполнителя в эластомерных композитах с помощью атомно-силового микроскопа // Вестник Пермского университета. Физика. 2018. № 1 (38). С. 36-45. doi: 10.17072/1994-3598-2018-1-36-45

Please cite this article in English as:

Garishin O. K., Izyumov R. I., Svistkov A. L. Features of the study of binder state near the filler particles in elastomeric composites using an atomic-force microscope // Bulletin of Perm University. Physics, 2018, no. 1 (38), pp. 36-45. doi: 10.17072/1994-3598-2018-1-36-45 